\title{
Designing Social Networks to Combat Fear of Missing Out
}

\author{
Aarif Alutaybi, John McAlaney, Angelos Stefanidis, Keith Phalp, Raian Ali \\ Bournemouth University, UK \\ \{aalutaybi, jmcalaney, astefanidis, kphalp, rali\}@bournemouth.ac.uk
}

\begin{abstract}
Fear of missing out, (hereafter referred to as FoMO), is increasingly becoming an issue of concern in relation to the use of Social Network Sites (SNSs). Despite its importance, the effects of FoMO continue to receive limited attention, while guidance on how SNSs design is responsible for developing should and combatting it, remains inadequate. In this position paper, we argue that dual responsibility of SNSs design. We report on initial results of a multiphase empirical study which was undertaken to examine the features of social networks that may contribute to triggering FoMO, and to explore how future SNSs can be designed to aid people manage their FoMO. The study involved three focus group sessions and a diary study. We argue that future SNSs shall support interaction styles and protocols and their agreement and adherence processes to enable people prevent and combat FoMO and present styles for doing that.
\end{abstract}

Fear of Missing Out, Social Networks Design, FoMO Countermeasures, Online Social Interaction

\section{INTRODUCTION}

As demonstrated extensively in social psychology individuals are driven to attempt to explain the actions of those around them, known as attributions (Hewstone and Jaspars, 1982). This has brought about a fundamental change in the locus of control in how people interact with their social group. In the past people may have attributed the frequency, or lack thereof, or interactions with their social group to the constraints of not being physically together. With the emergence of digital communication technologies and social media it is now almost always possible for individuals to communicate with their social group. As such the locus of control for social communication has changed from being partly externally determined to largely internally determined. However, the increased social interaction opportunities come at the cost of FoMO, which broadly expressed as a preoccupation with what is occuring online and how others are reacting, either positively or negatively, to someone's online presence and interactions (Beyen et al., 2016).

FoMO is defined as a "pervasive apprehension that others might be having rewarding experiences from which one is absent." It is characterised by the desire to stay continually connected with what others are doing (Przybylski et al., 2013). Social networks provide diverse information in real time about events, conversations and activities. While real time information allows people to remain abreast with new developments, it often facilitates FoMO behaviour by compelling people to check their social network activities frequently or stay online continuously to avoid missing out. FoMO demonstrates the desire of people who experience chronic deficits of psychological need satisfaction, to constantly engage with social networks, even when this occurs in unsuitable or dangerous situations such as while driving (Przybylski et al., 2013), or attending lectures (Alt, 2015; Turkle, 2011). It is important to note that as with many psychological disorders the core behaviour is not inherently problematic. Humans are a social species, and have evolved to exist within social groups that reinforce their group identity through the sharing of social information (Dunbar, 1996). As such there is a natural inclination to seek out social information, and to be concerned that your social group may exclude you if they perceive that you are not actively engaging with that group. Instead is with if the behaviour and associated cognitions become excesssive to the point of impacting on the individual's well-being.

Cheever et al. (2014) found evidence of negative influences, such as anxiety, when people could not access cyberspace. Anxiety constitutes a significant part of FoMO which is operationally defined as "the fears, worries and anxiety people may have in relation to being in (or out of) touch with events, experiences and conversations happening across their extended social circles" (Przybylski et al., 2013). In addition, Fox and Moreland (2015) suggested that FoMO is a reason why people feel pressured to continue using Facebook.

In this paper we argue and provide empirical evidence through a set of user studies that functional design of SNSs is partially responsibe for the manifestation of FoMO in users. We also sktech features and processes for designing SNSs in a way that aid individuals in managing their 
FoMO behaviour. Doing so would be consistent with the type of corporate social responsibility demonstrated within other industry that offer services to the public that have the potential to facilitate problematic behaviour, such as the alcohol and gambling industries.

The paper is structured as follows: Section 2 describes an exploratory study to get evidence regarding the association between the features of SNSs and FoMO. Section 3 shows initial results of the study. Section 4 describes the social interaction protocol governing FoMO. Section 5 presents the conclusions of the study and future work.

\section{METHOD AND RESEARCH SETTINGS}

This study aims to obtain evidence regarding the association between the features of SNSs and FoMO, and how SNSs can be designed to aid users to manage their FoMO behaviour. To achieve this, several qualitative studies were conducted.

The first study consisted of three gender balanced focus group sessions featuring twenty participants. Focus group sessions were used to give participants an opportunity to immerse themselves in the issue, discuss their opinions, and provide suggestions that may contribute to managing their FoMO. Each session included five to eight participants, aged between twenty and thirty years. Recruitment of the participants was done by placing an open call to a student forum, where individuals could self-nominate themselves to participate. Each group was given six scenarios with a set of prompts and a notepad to write their ideas on what actions might be appropriate, for each scenario, in order to manage FoMO.

The second study consisted of a diary study with twenty of the participants who participated in the focus group sessions. As part of this study, participants were asked to record their thoughts and feeling at least three times a day (morning, afternoon and evening) as they experienced FoMO. The justification for making multiple diary entries throughout the day stems from the fact that different SNSs are often used during the day and in different contexts, each of which may lead to FoMO occurring in different manners and to different degrees. Diary studies are also motivated by avoiding recall bias and ability to provide in-play data which are more expressive and contextualized. Participants were being given a link to diary form via the Evernote app and they were reminded to record in their diary by text messages. All participants in both studies self-declared to suffer FoMO in their usage of SNSs.

\section{RESULTS}

The initial analysis of the data focused on the features of social networks that could facilitate FoMO in users. The analysis was carried out using the Honeycomb classification of social networks features proposed (Kietzmann et al., 2011) as a template. Conversely, a number of features that may alleviate FoMO were also explored.

\subsection{SNSs Features and FoMO}

In this section, families of SNSs features and their relation to inciting FoMO will be discussed.

\section{- Group}

A group is defined as two or more individuals who are connected to each other through social relationships (Forsyth, 2006). Individuals participate in the group to satisfy their need to belong and, hence maintain their popularity. However, the group setting may facilitate FoMO by driving people to continually engage with the group's activities in order to maintain their presence and popularity in the group. For instance, participants mentioned that if they do not participate in the group their need to feel popular within the group cannot be satisfied, a clear example of fear of missing something. Within the group dynamic, there is a motivation to conform to the norms of the group (normative behaviour) in order to retain a sense of belonging to the group, and to maintain the desired level of perceived popularity and connectedness. For instance, one participant commented "I got so stressed because my friends wrote dozens of messages on WhatsApp group, but they did not say anything important. I do not have time to engage and I'm also not in the mood to interact but I have to read everything and reply in order not to lose my popularity among them".

\section{- Temporal events}

Temporal events are defined as events or opportunities which occur at a specific time and then expire or are removed. They may facilitate the triggering of fear of missing temporarily available information, as evidenced by participants who mentioned that temporal events trigger their FoMO and motivate them to interact with their social networks continually. A participant stated that "as Snapchat erases stories after sometime, I felt like l'd miss out on some celebrity' advice or product review, so I keep checking every few minutes."

\section{- Share}

Share is defined as the extent to which people exchange, distribute, receive, and share information including text, photos, audio and video. In this study, sharing appeared to trigger FoMO in which shared content may trigger either the fear of 
missing an opportunity or the fear of missing the ability of being popular. For example, one participant commented that: "My friend shared our trip pictures on Facebook that do not include me so I fear that I missed some interaction with him recently which led to him ignoring me."

\section{- Conversation}

Conversation represents the extent to which people communicate with others on social networks by exchanging messages, comments or chatting. This feature could facilitate FoMO by someone's inability to reply or being able to terminate a conversation. Thus, fear manifests itself as fear of missing empathy, fear of not leaving with a good impression, or fear of missing important messages which are part of a long conversation. For example: "My friend was talking to me about a personal matter and I couldn't stop using WhatsApp till 4:00 am as I feared my friend would think I do not want to listen to him." This reflects the social psychological concept of the norm of reciprocity, in which we feel obligated to return the effort that others have put into helping us (Cialdini, 2001).

\section{- Impression}

Impression is the feature that informs people how many individuals react to their social media shares, such as the number of 'Likes' or 'Retweets'. Participants highlighted the importance of this feature in measuring their popularity among peers when either receiving or not receiving a reaction. This type of feature relates to the fear of missing the ability to be popular or the ability to be seen as interesting when the number of reactions is unexpected. For example: "I did not receive likes and comments on pictures from number of friends as I expected. This made me anxious as I feared that I missed some interaction with them recently which led to them ignoring me". These concerns relate strongly to impression management, in which we are driven to manage how we are perceived by others and to monitor our success in achieving our desired social image (Goffman, 1959).

\section{- Trending}

Trending is the feature that identifies online content as having a high reputation. When people see content of high reputation, they become drawn to it and try to avoid missing it. As a result, time spent engaging with trending information may increase. For instance, one participant said that: "popular hash-tags make me feel I need to check every hour what is going on as I fear missing any updates."

\section{- Presence}

Presence allows individuals to become aware of the existence of other individuals from their profile or status. However, the presence feature contributes in triggering FoMO and, particularly, the fear of missing the ability to be popular. For example: "I have sent a WhatsApp message to a friend but she hasn't replied. However, I have seen that she was online. This made me anxious as I feared that I missed an earlier reply to her recently, which led to her ignoring me".

\subsection{FoMO Countermeasures}

This analysis has provided some initial suggestions of how SNSs can embed features to allow individuals to manage their feelings of FoMO. These suggestions or countermeasures to combat FoMo can be applied by individuals or social groups. The following section explains the initial FoMO countermeasures.

- Filter: is a technique that only shows the information that people are interested in. This technique can be applied when people fear missing out on information due to the existence of large volumes of it, as they are unable to connect or interact as they wish, for example "I am unable to use my phone as I am at work. Therefore when l'm on my break, I have 15 mins to read everything that has happened on all my social media during the time I have been at work. This can be hard as I have to prioritise, so that I have the most interactions with my friends but also find out what is going on as well. One suggested that "a useful way of getting over this situation is to filter and eliminate the spam on my timeline and news feed so that there is less to look at'.

- Priority list/ Importance Level: is a technique to allow people to specify the level of importance and priority of the various online activities they may be engaging in. While FoMO in general motivates people to stay online in order not to miss out on the interaction with others. This particular technique can alleviate some of the feelings of FOMO namely the fear of missing the opportunity to know someone else's impression. For example, one Psychology student said, "I uploaded some pictures on Instagram, so I was eagerly waiting for my friends to comment on them. I feared that if I did not check continuously I might miss my friends' comment on my post. I hope to cope better if I could get notifications in different tones, so that I can only check when they are about my post."

- Event recording: is a technique that records events while a person is offline to be viewed when they are online again. This technique can be helpful when people are unable to connect to SNSs and fear missing temporarily available information, 
such as stories on Instagram or Snapchat. For example: "I had an important task to do and couldn't check Instagram, so I worried about missing my friends' stories because postings are deleted after a while. Perhaps I can cope better if I can record the event."

- Set status and time: is a technique that allows people to set their current status and calendar availability. People usually encounter number of FoMOs when social groups do not interact with them as expected, for example the fear of not being popular or the fear of not being thought of to be interesting. However, setting a status and time may alleviate the level of this kind of FoMO. For instance, one Computing student said "It has been more than 5-6 hours since I upload my last post but until now, my friends haven't liked it or commented on it. I cannot figure out if they are ignoring me or they are busy. To minimise my FoMO, it would help if my friends could set their status accordingly to let me know that they are busy."

- Alternative notification: is a technique that allows people to receive notifications without having to use a social network, e.g., SMS or a vibration on their smartwatch. This technique may be useful for reducing compulsive behaviour of checking the notification on smartphone and then start checking other things. For example: "I usually check my phone continuously to see if I have any sort of message as I have in the habit of replying immediately. If there was a technique by which I could receive alternative notifications in different tones, I would be able to reduce my worry of missing out".

\section{TOWARDS FOMO-AWARE SNSs DESIGN}

As a part of our research, we argue that online social interactions among individuals can lead to experiencing FoMO. This results in increase the time of checking and thinking regarding the interactions that occur on SNSs. However, if individuals who are involved in these interactions have information regarding the situation of others this may raise the awareness and minimise FoMO. In this way the group members themselves can use this information to create a system that communicates their own ability and intention to interact with the group at any given moment. This could be achieved through a variety of techniques, including goal setting. This paper will give a brief explanation regarding the different modalities as following:
- Unilateral interaction protocol: it means that the protocol is set up by individuals themselves in order to alleviate their FoMO. This could include a self-set goal to only check social media a limited number of times a day. This goal could be made visible to other group members.

- Bilateral / reciprocal interaction protocol: it means that two or more individuals agree to set up their interaction protocol among them in a reciprocal manner, in order to alleviate mutual FoMO effects. Similar to the above this could be a mutual goal set by two individuals to limit their interactions. By doing so each individual is assured that the other is aware they will not respond immediately to any message or post.

- Collective interaction protocol: it means that the interaction protocol could be the responsibility of individuals who are involved in social interactions by setting up their own protocol. In this instance a group may collectively agree a protocol in which they all agree to disengage from their social media for a specific time period, such for example a group of students who need to focus their attention on studying for an exam.

However, the elements of the social interaction protocol in relation to alleviate FoMO such as the time availability for interaction or the situation of the individual have not been determined. In future, it is expected that the types of FoMO exhibited by users can be detected by SNSs. Based on the type of FoMO that individuals have, they may be given an awareness raising induction regarding FoMO and how this can be managed. This could build individuals' digital resilience in order to cope with FoMO when it is triggered. However, the specifications of the delivered resources are not yet known and individuals who are involved in creating those resources have not yet been defined.

\section{CONCLUSION AND FUTURE WORK}

FoMO is as a growing and important societal issue In this paper, the potential of SNSs features in triggering FoMO was identified and also a number of features that appear to be wanted by users in order to alleviate FoMO were discussed. The future work of this research is to obtain more evidence regarding the association between the features of SNSs and FoMO, and to obtain evidence regarding the effectiveness or the need for countermeasures. In addition, the research will investigate a different modalities and processes of designing and applying countermeasures. 


\section{REFERENCES}

Alt, D. (2015) College students' academic motivation, media engagement and fear of missing out. Computers in Human Behavior, 49, pp.111-119.

Beyens, I., Frison, E. and Eggermont, S., (2016) I don't want to miss a thing: Adolescents' fear of missing out and its relationship to adolescents' social needs, Facebook use, and Facebook related stress. Computers in Human Behavior, 64, pp.1-8.

Cheever, N.A., Rosen, L.D., Carrier, L.M. and Chavez, A. (2014) Out of sight is not out of mind: The impact of restricting wireless mobile device use on anxiety levels among low, moderate and high users. Computers in Human Behavior, 37, pp.290-297.

Cialdini, R. B. (2001) Influence: Science and Practice. Boston, MA: Pearson Education.

Dunbar, R. I. M. (1996) Grooming, gossip, and the evolution of language. Cambridge, Mass.: Harvard University Press.

Forsyth, D.R (2006) Group dynamics. Belmont, CA: Thomson-Wadworth.
Fox, J. and Moreland, J.J. (2015) The dark side of social networking sites: An exploration of the relational and psychological stressors associated with Facebook use and affordances. Computers in Human Behavior, 45, pp.168-176.

Goffman, E. (1959) The Presentation of Self in Everyday Life. New York: Anchor Books.

Hewstone, M., \& Jaspars, J. M. F. (1982) Intergroup relations and attribution processes. In H. Tajfel (Ed.), Social Identity And Intergroup Relations (pp. 99 - 133). Cambridge: Cambridge University Press.

Kietzmann, J.H., Hermkens, K., McCarthy, I.P. and Silvestre, B.S. (2011) Social media? Get serious! Understanding the functional building blocks of social media. Business horizons, 54(3), pp.241251.

Przybylski, A.K., Murayama, K., DeHaan, C.R. and Gladwell, V. (2013) Motivational, emotional, and behavioral correlates of fear of missing out. Computers in Human Behavior, 29(4), pp.18411848. 